\title{
Growth Hormone: The Body Knows when to Quit
}

\author{
Charles J Malemud ${ }^{\star}$ and Joanne D Denko
}

Professor of Medicine and Anatomy, Division of Rheumatic Diseases, Case Western Reserve University School of Medicine \&University Hospitals Cleveland Medical Center, Foley Medical Building 2061 Cornell Road, Room 207,Cleveland, Ohio, USA

*Corresponding Author: Malemud CJ, Professor of Medicine and Anatomy, Division of Rheumatic Diseases, Case Western Reserve University School of Medicine \& University Hospitals Cleveland Medical Center, Foley Medical Building, 2061 Cornell Road, Room 207, Cleveland, Ohio 44106-5076, USA, Tel: 216-844-7846; Fax: 216-844-2288; E-mail:cjm4@cwru.edu

Received date: November 5, 2016; Accepted date: December 2, 2016, Published date: December 5, 2016

Copyright: (c) 2017 Malemud CJ, et al. This is an open-access article distributed under the terms of the Creative Commons Attribution License, which permits unrestricted use, distribution, and reproduction in any medium, provided the original author and source are credited.

\section{Opinion}

A recent advertisement in a popular magazine touted human growth hormone $(\mathrm{HGH})$ for alleged anti-aging properties. In that regard, the advertisement contends that increasing the level of $\mathrm{HGH}$ secretion acts to blunt the aging process. This viewpoint is apparently formulated on the basis of the "natural decline of human growth hormone from ages 21 to 61 " (the average age at which there is only a trace left in the body). Therefore, the decline in HGH is purported to be the main reason why aging occurs and according to this advertisement "fails to regenerate to its 25 year-old biological age".

Because research in this area showed that the major components of the aging process include altered cognition, increased frailty coupled to a reduction in energy, as well as various constitution symptoms related to muscle and joint function such as pain and reduced activity, the issue of whether HGH is associated with these changes suggests that its use as a supplement non-prescription agent would be highly germane in terms of improving the quality of daily living.

Thus, the main purpose of this opinion article is to challenge the underlying assumption that supplementation using human growth hormone receptor (GHR) which promotes increased release of $\mathrm{HGH}$ from the pituitary gland is a prudent approach for increasing longevity put another way, would GHR supplementation constitute an anti-aging strategy? Consistent with the viewpoint that supplemental GHR promotes longevity, the advertisement in question also stated that GHR is a "natural releaser with no known side-effects but unlike a synthetic version of GHR, has no known drug interactions." Moreover, the advertisement asserted that "continued use of GHR will make a radical difference in your health." Finally, this advertisement stated that $\mathrm{HGH}$ is known to relieve symptoms of many diseases, including fibromyalgia, among other chronic medical conditions, which led to the assertion that the promotion of $\mathrm{HGH}$ release via supplemental GHR would ameliorate these symptoms.

In order to assess systematically the extent to which the GHR supplement (commercially available from BIE Health Products, Amherst, New York) would perform in the way it is claimed to act it is important to assess in a critical manner just how growth hormone is regulated in animals and normal humans and whether or not increasing HGH secretion is, in fact, a rational approach for relieving chronic pain which is a major component of medical conditions such as the fibromyalgia syndrome. In that regard, fibromyalgia is cited at the end of the advertisement as a medical condition responsive to HGR-mediated HGH release. Another issue which we will discuss is the extent to which exogenous HGH produces an anti-aging effect.

Beginning in the early 1990's Denko et al. at Case Western Reserve University (CWRU) School of Medicine, Cleveland, Ohio, initiated a series of investigations which became the seminal studies that indicated that $\mathrm{HGH}$ appeared to play a significant role in the progression of certain musculoskeletal diseases based on the evidence that elevated levels of serum HGH were produced in these disorders. Thus, the findings from many of these studies in patients with osteoarthritis (OA) [1] as well as additional results from studies performed by the CWRU group and other investigators indicating a strong association between serum HGH levels and musculoskeletal conditions were eventually summarized in a review of the published literature on this subject [2].

HGH is a 191-amino-acid-containing neuropeptide which is primarily involved in skeletal long bone growth and development [3] as well as acting as a critical component for chondrocyte maturation [4-6]. Importantly, evidence accumulated over the last 25 years or so has persuasively shown that HGH can be found in association with the state of chronic inflammation [6].

In that regard, data directly relevant to the thrust of using GHR supplementation to improve $\mathrm{HGH}$ release are the results of a classic study by Waters et al. [7] who demonstrated that HGH activity was directly linked to activation of the GHR. Thus, this is the metabolic step which initiates HGH-mediated signaling and routinely results in several downstream cellular effector and activation events, many of which are essential either to cell survival, programmed (i.e. controlled) cell death or other forms of cell-killing. For example, the loss of HGHmediated signal transduction which occurs in signal transducers and activators of transcription-5 (STAT-5)-null transgenic mice causes a significant suppression of postnatal bone development [8]. Moreover, developmental abnormalities in the bones of these STAT- 5 null mice are comparable to abnormalities in those mice in which GHR mutations have been introduced [8]. Of note, the results of these elegant studies also provided direct evidence linking the function(s) of HGH to GHR activity.

Although it would require a separate commentary to address adequately the extent to which an association exists between aging and serum levels of $\mathrm{HGH}$, the most fundamental conclusions drawn from many years of active basic and clinical research in this area can be summarized as follows: In the 1990's, researchers found that both aging and HGH deficiency are related to one another. In that regard, aging was characterized by a reduced level of serum HGH and protein synthesis, decreased lean body and bone mass as well as increased body fat [9]. Moreover, in healthy individuals, the level of spontaneous as well as stimulated $\mathrm{HGH}$ secretion are significantly decreased with advanced aging [9]. However, these findings must now be re-evaluated in view of more recent findings reported by Bartke et al. [10] who showed that reduced levels of $\mathrm{HGH}$ and insulin-like growth factor-1 (IGF-1) activity are associated with delayed or a "slower" rate of aging. 


\title{
(10.4)
}

\author{
(2017)
}

uction in frailty,

Thus, a lower level of HGH was correlated with a reduction in frailty,
age-related diseases and a decline in functional status, BUT most critically, in an extension of longevity. Therefore, it appears from summarizing these human studies that stimulation of HGH secretion by ingesting supplemental HGR could actually reduce longevity. Of note, many of the findings relating $\mathrm{HGH}$ levels in humans as a function of age have been sustained from results of studies of GH levels in aging and in lifespan in which experimental animal models of aging were employed $[11,12]$.

In addition to the direct effects of HGH on human metabolism, it is of utmost importance to emphasize that an association also exists between HGH activity and IGF-1 [2,4] because IGF-1 production is generally dependent on $\mathrm{HGH}$ in what is termed the HGH/IGF-1 somatotropic axis [10]. Thus, this aptly named HGH/IGF-1 paracrine axis constitutes a positive feedback loop for determining the level of HGH biological activity. Conversely, somatostatin, a 14-amino-acid polypeptide which is widely distributed throughout the central nervous system as well as being produced by cells in peripheral tissues was shown to be the endogenous inhibitor of $\mathrm{HGH}$ release from the pituitary gland [13].

Now that we have briefly reviewed the cellular signaling mechanism attributed to the biological activities of $\mathrm{HGH}$, it is important to review the status of serum levels of HGH, IGF-1 and somatostatin in various musculoskeletal diseases reported in the published literature as archived in the US National Library of Medicine's, PubMed database, in an attempt to predict the extent to which increasing the level of circulating HGH via GHR supplementation would be expected to alleviate the clinical symptoms of some of these musculoskeletal disorders.

We begin with a short review of the status of serum HGH, IGF-1 and somatostatin in a few rheumatic diseases, including fibromyalgia. Previous studies in human OA patients [1] have been most revealing with respect to how serum levels of HGH, IGF-1 and insulin are associated with this musculoskeletal disease of synovial joints. Furthermore, we also now appreciate the extent to which synovial inflammation, a driving force in the progression of $\mathrm{OA}$, is often dependent on the stage of OA pathology. Since HGH levels (or levels of other factors such as insulin and IGF-1) vary diurnally it is incumbent that blood be collected for evaluation at the same time, preferably in early morning when HGH levels are generally at basal levels. Otherwise the diurnal variations will confound the effort to track the serum levels of these factors. Thus by keeping these critical issues in mind, Denko et al. [1] showed that serum HGH and insulin levels were significantly elevated in OA patients compared to a control non-OA group, whereas IGF-1 levels were lower.

Furthermore, in rheumatoid arthritis (RA) patients where chronic, systemic inflammation is a hallmark and an essential characteristic of this disease process, serum HGH was also significantly elevated [14]. In fact, the ratio of serum HGH to somatostatin was significantly increased in RA patients by virtue of the markedly reduced level of serum somatostatin in these patients [14]. Therefore, the primary conclusion drawn from the results of this study was the finding of a strong association between chronic systemic inflammation, elevated serum HGH levels and lower somatostatin levels.

Because chronic inflammation is a clinical component of RA and progressive OA, Denko and Malemud [15] measured the serum levels of HGH, IGF-1 and insulin in patients with normal serum glucose levels and a clinically confirmed diagnosis of fibromyalgia.

Fibromyalgia is considered by most clinicians and basic researchers to be a musculoskeletal condition which is essentially devoid of chronic inflammation. The findings in that study were that serum HGH and insulin, but not IGF-1, were elevated in fibromyalgia patients, suggesting that basal serum HGH and fasting insulin levels were a surrogate biomarker for assessing the clinical course of fibromyalgia.

However, the results of some studies of fibromyalgia patients produced countervailing evidence to that reported by Denko and Malemud [15]. In that regard, Jones et al. [16] reported normal pituitary function in fibromyalgia patients, where levels of serum HGH did not correlate with IGF-1 as would be expected if IGF-1 is solely HGH-dependent. Thus, in that study daily administration of recombinant human HGH normalized IGF-1 levels while also reducing the symptoms of fibromyalgia [16]. However, Tander et al. [17] showed no significant difference between fibromyalgia patients and a control group with respect to serum HGH, IGF-1 or insulin levels. Of note, the level of serum ghrelin (a gastric hormone related to hunger) was significantly lower in fibromyalgia patients compared to controls, although the significance of this secondary end-point remains to be elucidated.

Despite some results which did not appear to confirm those reported by Denko and Malemud [15], another series of clinical studies initiated by Cuatrecasas et al. [18-20] were designed to determine the extent to which exogenously-administered $\mathrm{HGH}$ provided to fibromyalgia patients with low IGF-1 levels would be of clinical benefit in the treatment of fibromyalgia $[19,20]$. The most recent clinical trial in this area was conducted under rigorous, randomized, doubleblinded, controlled conditions which showed that fibromyalgia patients, who were concomitantly receiving stable doses of amitriptyline, selective serotonin reuptake inhibitors and tramadol as part of their treatment regimen, and who also presented with the lowest levels of HGH and IGF-1, were most responsive clinically to supplemental HGH therapy [21]. However, a recent analysis by Blumenthal and Malemud [22] re-evaluated the data from that clinical trial [21] where the primary end-point measured in response to treatment with exogenous HGH was a reduction in the number of fibromyalgia tender points and concluded that, in fact, no statistically significant difference could be found between the HGH-treated and the placebo arm of the clinical trial in either primary or secondary end-points. Therefore, it can be concluded that the administration of HGH or supplemental HGR to alleviate the chronic pain of fibromyalgia remains controversial, to say the least.

In discussing the significance of elevated serum levels of $\mathrm{HGH}$ in OA patients, Denko and Malemud [23] proposed that OA should be considered a systemic metabolic disturbance rather than merely a focal degeneration of synovial joint articular cartilage, the growth of osteophytes, and sclerotic thickening of subchondral bone. In addition, in the context of the elevated levels of serum HGH in OA patients, these HGH levels could be interpreted as being toxic when HGH is transported from the peripheral circulation to the joint synovial fluid, where these elevated levels of HGH may alter articular cartilage and subchondral bone homeostasis [24].

Most importantly, the regulated secretion of $\mathrm{HGH}$ is a fundamental property of this hormone. Thus, the level of HGH remains low after growth plate maturation, and in so far as homeostasis is concerned, HGH levels continue to be low. However serum HGH appears to rise in conjunction primarily with the progression of inflammatory diseases where $\mathrm{HGH}$ may be associated with increased controlled cell death. Thus, we assert that low levels of serum HGH as found in older 
individuals during the aging process is consistent with the nostrum that "the body knows when to quit."

In conclusion then we ask what possible rationale can be provided for increasing the level of HGH with supplemental GHR in order to "slow down" the aging process and improve well-being, if as a consequence of normal aging, HGH is produced only in small amounts [9] as was found to be the case in older individuals? Once again, our viewpoint is also supported by results of studies in experimental animals which revealed that GHR gene-disrupted mice were shown to have an extended lifespan [25], and where lifespan was not altered in mice expressing a GH antagonist [26].

\section{References}

1. Denko CW, Boja B, Moskowitz RW (1990) Growth promoting peptides in osteoarthritis: insulin, insulin-like growth factor-1, growth hormone. J Rheumatol 17: 1217-1221.

2. Denko CW, Malemud CJ (2005) Role of the growth hormone/insulin-like growth factor-1 paracrine axis in rheumatic diseases. Semin Arthritis Rheum 35: 24-34.

3. Yakar S, Isaksson O (2016) Regulation of skeletal growth and mineral acquisition by the GH/IGF-1 axis: lessons from mouse models. Growth Horm IGF Res 28: 26-42.

4. Malemud CJ (1993) The role of growth factors in cartilage metabolism In: Rheum Dis Clin North Am. Moskowitz RW (Editor), Philadelphia, Saunders, 19: 569-580.

5. Reinecke M, Schmid AC, Heyberger-Myer B, Hunziker EB, Zapf J (2000) Effect of growth hormone and insulin-like growth factor-1 (IGF-1) on the expression of IGF-1 messenger ribonucleic acid and peptide in rat tibial growth plate and articular chondrocytes in vivo. Endocrinology 141 2847-2853.

6. Pap T, Distler O (2005) Linking angiogenesis to bone destruction in arthritis. Arthritis Rheum 52: 1346-1348.

7. Waters MJ, Hoang HN, Fairlie DP, Pelekanos RA, Brown RJ (2006) New insights into growth hormone action. J Mol Endocrinol 36: 1-7.

8. Woefle J, Chia DJ, Rotwein P (2003) Mechanisms of growth hormone (GH) action. Identification of conserved Stat 5 binding sites that mediate $\mathrm{GH}$-induced insulin-like growth factor-1 gene activation. J Biol Chem 278: 51261-51266.

9. Corpas E, Harman SM, Blackman MR (1993) Human growth hormone and human aging. Endocr Rev 14: 20-39.

10. Bartke A, List EO, Kopchick JJ (2016) The somatotropic axis and aging: benefits of endocrine defects. Growth Horm IGF Res 27: 41-45.

11. Cater CS, Ramsey MM, Sonntag WE (2002) A critical analysis of the role of growth hormone and IGF-1 in aging and lifespan. Trends Genet 18 295-301.
12. Berryman DE, Christiansen JS, Johannsson G, Thorner MO, Kopchick JJ (2008) Role of the GH/IGF-1 axis in lifespan and healthspan: lessons from animal models. Growth Horm IGF Res 18: 455-471.

13. Kreienkamp HJ (1999) Molecular biology of the receptors for somatostatin and corticostatin. In: Richter D, Editor. Regulatory Peptides and Cognate Receptors 26: 215-237.

14. Denko CW, Malemud CJ (2004) The serum growth hormone to somatostatin ratio is skewed upward in rheumatoid arthritis patients. Front Biosci 9: 1660-1664.

15. Denko CW, Malemud CJ (2005) Serum growth hormone and insulin, but not insulin-like growth factor-1 levels are elevated in patients with fibromyalgia syndrome. Rheumatol Int 25: 146-151.

16. Jones KD, Deodhar P, Lorentzen A, Bennett RM, Deodhar AA (2007) Growth hormone perturbation in fibromyalgia: a review. Semin Arthritis Rheum 36: 357-379.

17. Tander B, Atmaca A, Aliyazicioglu Y, Canturk F (2007) Serum ghrelin levels but not GH, IGF-1 and IGFBP-3 levels are altered in patients with fibromyalgia syndrome. Joint Bone Spine 74: 477-481.

18. Cuatrecasas G (2009) Fibromyalgic syndromes: could growth hormone therapy be beneficial? Pediatr Endocrinol Rev 6 Suppl 4: 529-533.

19. Cuatrecasas G, Alegre G, Casanueva FF (2014) GH/IGF1 axis disturbances in the fibromyalgia syndrome: is there a rationale for $\mathrm{GH}$ treatment? Pituitary 17: 277-283.

20. Cuatrecasas G, Riudavets C, Güell MA, Nadal A (2007) Growth hormone as concomitant treatment in severe fibromyalgia associated with low IGF-1 serum levels. A pilot study. BMC Musculoskelet Disord 8: 119.

21. Cuatrecasas G, Alegre C, Fernandez-Solà J, Gonzalez MJ, GarciaFructuoso F, et al (2013) Growth hormone treatment for sustained pain reduction and improvement in quality of life in severe fibromyalgia. Pain 153: $1382-1389$.

22. Blumenthal DE, Malemud CJ (2016) Recent strategies for drug development in fibromyalgia syndrome. Expert Rev Neurotherapeut 14: 1-5.

23. Denko CW, Malemud CJ (1999) Metabolic disturbances and synovial joint responses in osteoarthritis. Front Biosci 4: d686-d693.

24. Denko CW, Boja B, Malemud CJ (2003) Intra-erythrocyte deposition of growth hormone in rheumatic diseases. Rheumatol Int 23: 11-14.

25. Young JA, List EO, Kopchick JJ (2016) Deconstructing the growth hormone receptor (GHR): physical and metabolic phenotypes of tissuespecific GHR gene-disrupted mice. Prog Mol Biol Transl Sci 138: 27-39.

26. Coschigano KT, Holland AN, Riders ME, List EO, Flyvbjerg A, et al. (2003) Deletion, but not antagonism, of the mouse growth hormone receptor results in severely decreased body weights, insulin, and insulinlike growth factor I levels and increased lifespan. Endocrinology 144: 3799-3810. 